\title{
EXPLORING THE INFLUENCE OF LIGHT AND COGNITIVE LOAD ON PUPIL DIAMETER IN DRIVING SIMULATOR STUDIES
}

\author{
Oskar Palinko \& Andrew Kun \\ University of New Hampshire \\ Durham, New Hampshire, USA \\ E-mail: oskar.palinko@unh.edu
}

\begin{abstract}
Summary: Pupil diameter can be used as a physiological measure of cognitive load in driving simulator studies. However, pupil size depends on both cognitive load and lighting conditions. In order to accurately estimate cognitive load these two effects must be separated. In our study we introduce illumination only, cognitive only and combined tasks. Based on these we decouple the two effects on pupil diameter and we design a predictor of the pupil's reaction to light which can be used to estimate changes in pupil diameter that are due to cognitive load.
\end{abstract}

\section{INTRODUCTION}

With the proliferation of in-car electronic devices such as cell phones, personal navigation devices, and in-car entertainment systems, drivers' cognitive resources are taxed as perhaps never before. Designers of in-car user interfaces utilize performance, physiological and subjective measures in order to estimate the cognitive load introduced by these interfaces. One of the physiological measures is pupil diameter. Pupil diameter is of interest due to the fact that when people are faced with a challenging cognitive task, their pupils dilate. This phenomenon is called the Task Evoked Pupillary Response (TEPR) (Beatty, 1982; Klingner et al., 2008).

However, cognitive load is certainly not the only factor influencing pupil diameter. The pupil's primary task is to regulate the amount of light falling on the retina. When there is too much light, the sphincter pupillae muscle group will contract the pupil, to reduce influx. When there is not enough light, the pupil will dilate by the activation of the dilator pupillae muscle group. Our long term goal is to design an algorithm to use pupil diameter as a measure of cognitive load in driving simulator experiments, even when the light reaching the pupil from the simulator screen changes over time. In this study we hypothesize (H1) that it is possible to decouple the TEPR and the pupil's light reflex for subjects scanning static images in a driving simulator. We also hypothesize (H2) that under such conditions it is possible to predict pupil diameter changes due to the light reflex and subtract them from the overall changes in order to estimate the TEPR.

Pupil diameter can be measured using eye trackers. Using a remote eye tracker Klingner et al. (2008) successfully estimated cognitive load in a desktop environment, while our group did so in a driving simulator (Palinko et al., 2010). However, neither study explored the interaction between cognitive load and the pupil's light reflex, opting instead to maintain near-constant lighting. For example, in our driving simulator study we confirmed that the average illumination of the simulator screen for each simulation frame was within $+/-5 \%$ of the overall mean calculated over the entire length of the experiment. Based on this calculation we made the assumption that the light reflex did not significantly influence pupil diameter. In contrast, in this 
paper we make no such assumptions and explore how changes in cognitive load and lighting interact in influencing pupil diameter.

Pomplun and Sunkara (2003) demonstrated the effects of cognitive load and lighting on the average pupil diameter. The authors suggest subtracting the predicted pupil diameter for a given lighting level from the overall pupil diameter, resulting in diameter changes that are presumably due to changes in cognitive load only. We also suggest taking this approach, but in contrast to Pomplun and Sunkara, we investigate the pupil's entire time-domain response, not only the timeaveraged response for different levels of cognitive load and lighting. We expect that this approach will allow us to track rapid cognitive load-induced changes.

Asano et al. (1994) also explored the pupil’s time-domain response to changes in lighting, but without manipulating cognitive load. They found that a neural network model of the pupil's response outperforms a linear model. Van Orden et al. (2001) also used neural networks to predict the pupil's response to changes in cognitive load, but without manipulating lighting. We present a model that uses two separate linear transfer functions, and a saturation element, to predict dilation and contraction in response to lighting changes. With this model we demonstrate that it is possible to predict the light reflex, account for its effect on pupil diameter, and thus track cognitive load-induced changes in pupil diameter.

\section{METHOD}

\section{Equipment}

The study was conducted in a DriveSafety DS-600C high fidelity driving simulator (Figure 1a). Although no driving was done for this study the simulator was used to project images, play sounds and to create a realistic driving environment. Gaze direction and pupil size were recorded using a SeeingMachines faceLAB 5.01 remote eye tracker. The eye tracker was mounted in the simulator on top of the dashboard in front of the driver (Figure 1b). The eye tracking system estimates the pupil diameter based on an ellipse fitting algorithm.

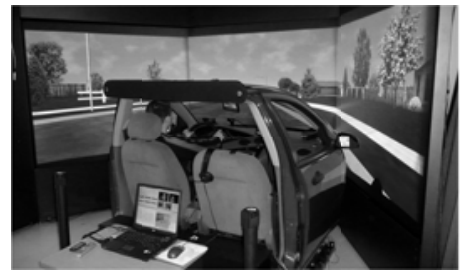

Figure 1a. Driving simulator

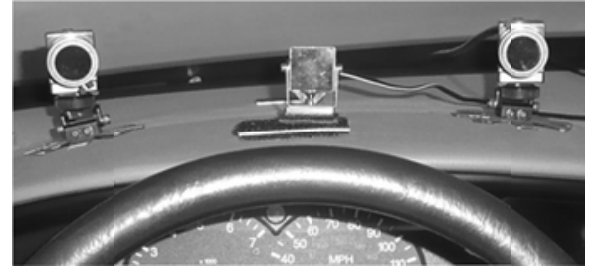

Figure 1b. Eye tracker on dashboard

\section{Tasks}

The subjects were asked to complete three different tasks: Illumination, Aural Vigilance and Combined Task. Each of these was preceded by a minute of training and followed by filling out the NASA-TLX form. The order of the tasks was counterbalanced between subjects.

The Illumination Task (IT) consisted of looking at a static simulated image of three trucks of different shades of gray (Figure 2). This image was projected on the front screen of our simulator 
while the other screens (left, right, rear, side view) were turned off. The left truck's area was nearly black (referred to as black, from now on) with $10 \%$ of the projector's maximum brightness (1.3 lux, measured with Velleman DVM1300 light meter). The middle truck's area was gray with $50 \%$ brightness (31.6 lux), while the right truck was nearly white (referred to as white, from now on) with $90 \%$ of brightness (193.1 lux). While the trucks were without color, their environment was naturally colored. The image represented a city intersection with traffic lights, thus simulating waiting at a red light. Subjects were instructed to fixate their gaze on a small target that was positioned on one of the three trucks (on gray truck in (Figure 2). The target was presented in white on the black and gray trucks, while it was gray on the white truck.

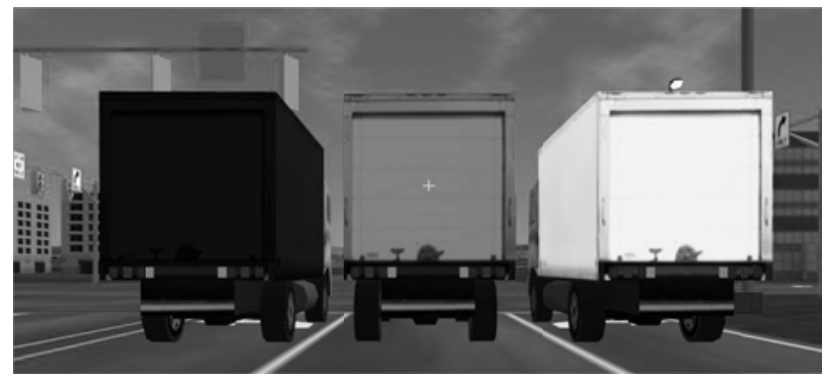

Figure 2. The image of three trucks presented during the Illumination and Combined Tasks

The task started with the target on the middle (gray) truck, where it stayed for 15 seconds, providing time for the eyes to adapt. After this period the target moved from one truck to another 12 times, remaining on each of the trucks for 9 seconds. The order in which the target moved between trucks was kept constant for all subjects. Subjects fixated on each truck 4 times and they experienced each transition between brightness levels (black->gray, white->black, etc.) 2 times.

The Aural Vigilance Task (AVT) was adapted from Klingner et al. (2008). It consisted of listening to a pre-recorded male voice counting from 1 to 18 repeatedly (after 18 it would restart at 1$)$. Subjects were told that every $6^{\text {th }}$ number $(6,12$, and 18$)$ might be out of order. This is illustrated in Figure 3, , where the $6^{\text {th }}$ number is out of order but the $12^{\text {th }}$ is not. Subjects were instructed to press a button on the steering wheel if they detected an out-of-order number. The task induced increased cognitive load at every $6^{\text {th }}$ number, as this was the point where subjects had to identify if a number was out of order. This increase in cognitive load triggered the TEPR, manifesting in a short dilation of the pupil. The 1-18 counting sequence was repeated 4 times, thus subjects had to pay attention to $4 \times 18 / 6=12$ possible errors. The location of errors was randomly selected but kept constant for all subjects. Out of the 12 possible locations the counting sequence included an error in 6. The time between the start of each number was $1.5 \mathrm{sec}$. During the AVT the simulator screen was set to gray, keeping lighting constant.

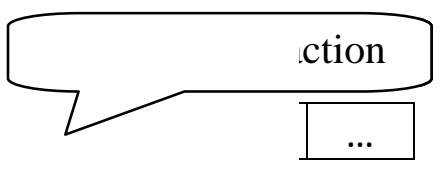

Figure 3. Counting sequence in the Aural Vigilance and Combined Tasks 
In the Combined Task (CT) subjects performed the IT and the AVT in parallel. They followed the target with their gaze while listening to the counting sequence and operating the button on the steering wheel. This task produced an interaction between the pupil light reflex and the TERP.

\section{Subjects}

The experiment was completed by 12 male college students, all native speakers of English. We offered a gift card compensation of $\$ 10$ for completing the experiment and an additional \$5 for good performance, which was always awarded. The subjects’ mean age was 20.6 years. A condition of participation was not to wear glasses, because of the possible interference of the infra-red illuminator's reflection off the glasses with pupil tracking.

\section{RESULTS}

\section{Illumination Task}

The mean pupil diameter change (MPDC) for a subject is defined as the average pupil diameter in a given number of time periods minus the mean value of the pupil diameter in the whole experimental run. The overall mean is subtracted because people's pupils are of different sizes. We calculated the MPDC for each subject and for each level of lighting intensity. Figure 4 shows the average MPDC for all subjects for the cases when they were looking at the black, gray and white trucks respectively. The results were analyzed using one-way repeated measures ANOVA. Since sphericity could not be assumed, the Greenhouse-Geisser correction was applied. The difference between the MPDC for the black, gray and white trucks was found to be statistically significant with $F(1.26,13.87)=64.9, p<0.001$. This finding demonstrates that the pupil size will depend heavily on the illumination of a limited area around the gaze direction.

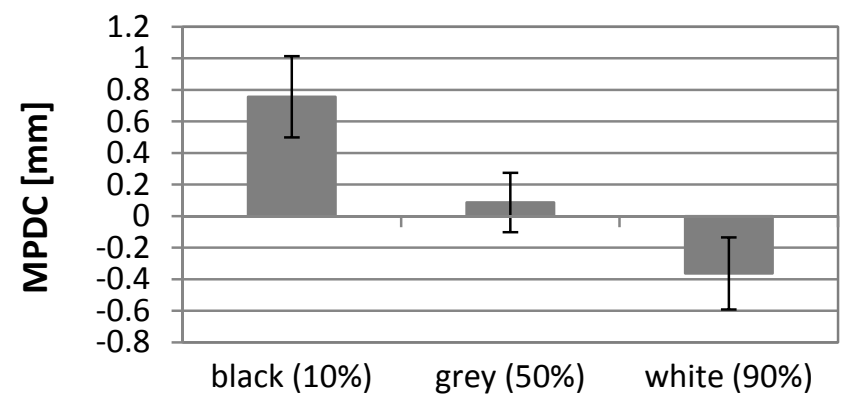

Figure 4. Mean pupil diameter change while looking at trucks of different brightness (+/- 1 SD)

Next, we analyzed pupil response to change in lighting intensity as subjects' glances switched from truck to truck. We averaged the pupil diameter for all subjects. Figure 5a shows the pupil's reaction to a switch to a brighter period (pupil contraction), while Figure 5b shows the switch to a darker period (pupil dilation). In both figures lighting intensity changes at $t=0 \mathrm{~s}$. 


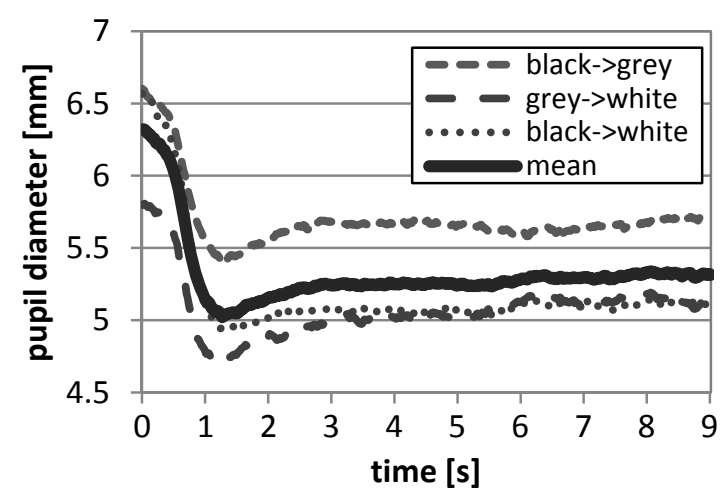

Figure 5a. Response to onset of a brighter period

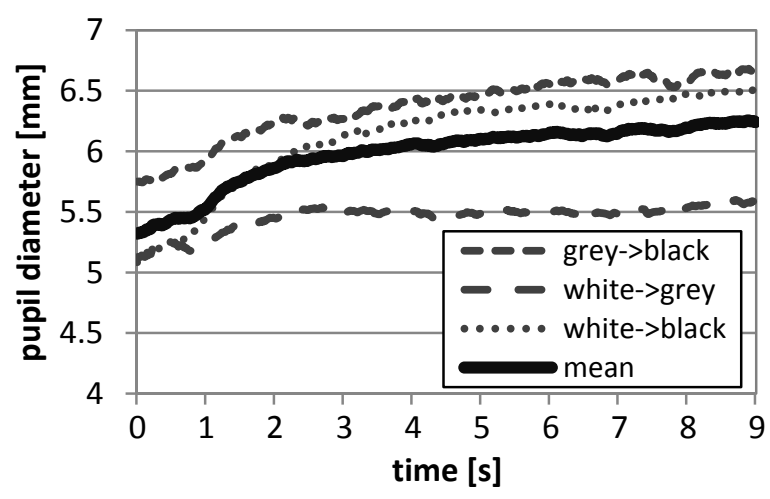

Figure 5b. A darker period

Note that the two graphs in Figure 5 are very different in nature. This is because of the different muscle groups involved in contraction and dilation. The reaction to a bright light must be quicker to protect the retina from overexposure. On the other hand reaction to darkness is more gradual, because there is no need to protect the retina from darkness. Also note that pupil size depends both on the previous and current level of lighting intensity.

\section{Aural Vigilance Task}

In order to evaluate how pupil diameter changes through the counting sequence we averaged pupil responses for each subject and for each six-number period. Note that every sixth number could be out of order, so the pattern of pupil dilation and contraction repeats. As shown in Figure 6, and in agreement with Klingner et al. (2008), we found a prominent peak in the pupil signal during the utterance of "six" which also represents the time when "twelve" and "eighteen" were uttered. The peak is due to subjects paying increased attention as they expect an error. Its existence indicates that pupil diameter patterns could be used to estimate cognitive load.

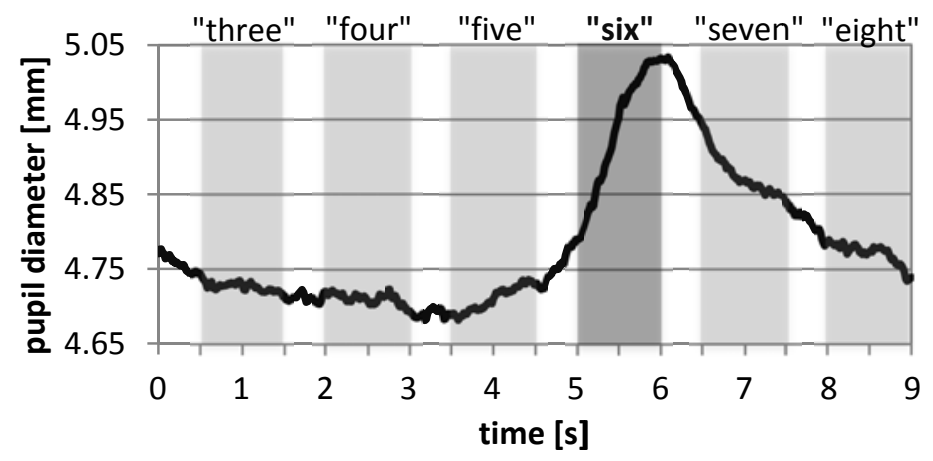

Figure 6. Pupil diameter signal for Aural Vigilance Task over all subjects

\section{Combined Task}

Figure 7a shows the average reaction to illumination increase for the Combined Task and Illumination Task (data for the IT was introduced in Figure 5a). Figure 7b shows the result of subtracting the IT mean from the CT mean. Note that the shape of this difference graph very much resembles that of the Aural Task (Figure 6). Also, the diameter difference between the 
"three" and "six" segments is in the 0.3-0.4 mm range in both figures. Thus, in this situation it was possible to estimate the cognitive load from the difference between the two signal averages.

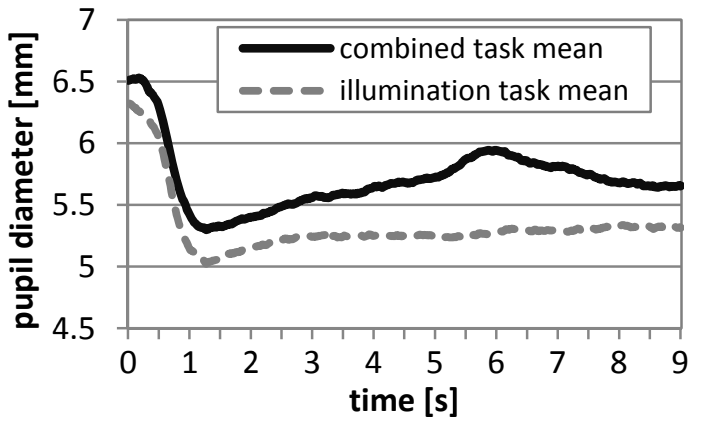

Figure 7a. Mean reaction to increase in illumination for CT and IT

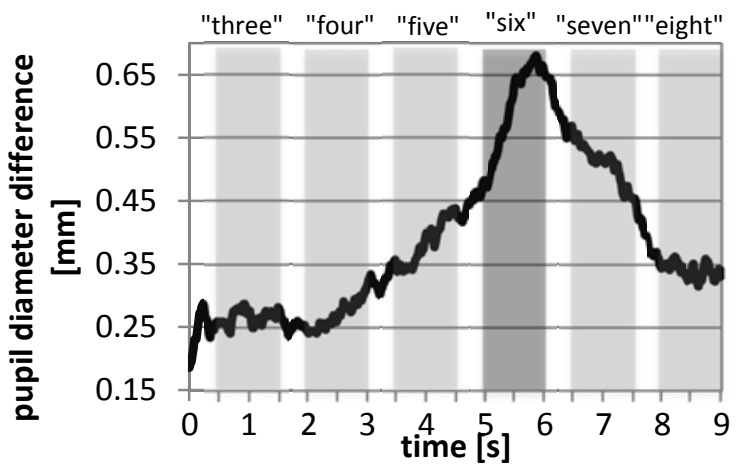

Figure 7b. Difference between these two

\section{Prediction}

The results in the previous tasks encouraged us to design a predictor of the pupil size reaction to illumination, with the goal to subtract the predictor's output from the pupil signal and thus end up with an estimate of TEPR alone. Based on observations of the Illumination and Combined Tasks we set forth the predictor model in Figure 8.

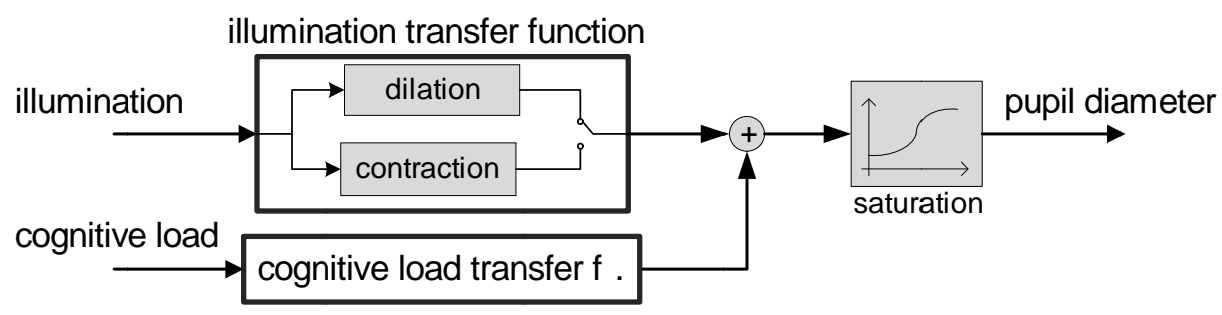

Figure 8. Block diagram model of the influence of illumination and cognitive load on pupil diameter

The illumination transfer function consists of two elements; because the pupil's reactions to increase and decrease in light have different characteristics (see Figure 5). The model also contains a saturation element, because the pupil has its minimum and maximum physical limits. In our prediction only the illumination transfer function was identified, while the cognitive load transfer function will be defined in future studies. Parameter estimation of the dilation and contraction transfer functions' numerator and denominator was performed using Matlab's arx function. The targets of this estimation were the mean contraction and dilation step response curves from Figure 5. This initial model of the system approximates the pupil's reaction to light quite well in some situations, as for example for Subject 7, as shown in Figure 9. 

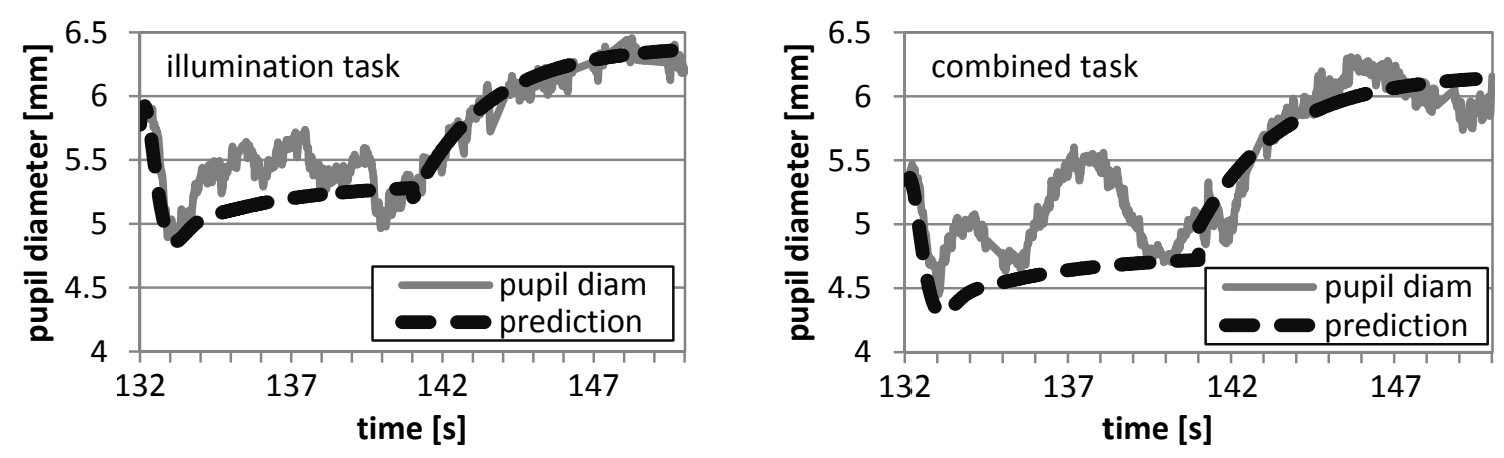

Figure 9. Pupil diameter and predictor output for Subject 7 during the Illumination Task (left) and Combined Task (right)

Figure 9 presents two examples of Subject 7 shifting his gaze from a darker to a lighter truck at $\mathrm{t}=132 \mathrm{~s}$ and then to a darker truck 9s later at $\mathrm{t}=141 \mathrm{~s}$. The prediction follows the Illumination Task pupil signal more precisely, than for the Combined Task. In the latter case, there are bumps in the pupil signal at $\mathrm{t}=137 \mathrm{~s}$ and $\mathrm{t}=146 \mathrm{~s}$ corresponding to locations of increased cognitive load. Notice that while the pupil size is smaller (lighter truck) the cognitive effects (manifesting in bumps in pupil diameter) are more pronounced than when the pupil size is larger (darker truck). This is due to the physical limits of the size of the pupil. In these border cases the simple addition of signals is distorted by saturation (see model in Figure 8).

Finally, we subtracted the predictor signal from the pupil signal during the Combined Task for all subjects. The results were averaged over all six number periods and over all subjects. The resulting graph in Figure 10 is very similar to the results of the Aural Vigilance Task (see Figure 6). This similarity is an indication that the predictor is able to discern light reflex and cognitive load effects on pupil size when the results are averaged over many cases.

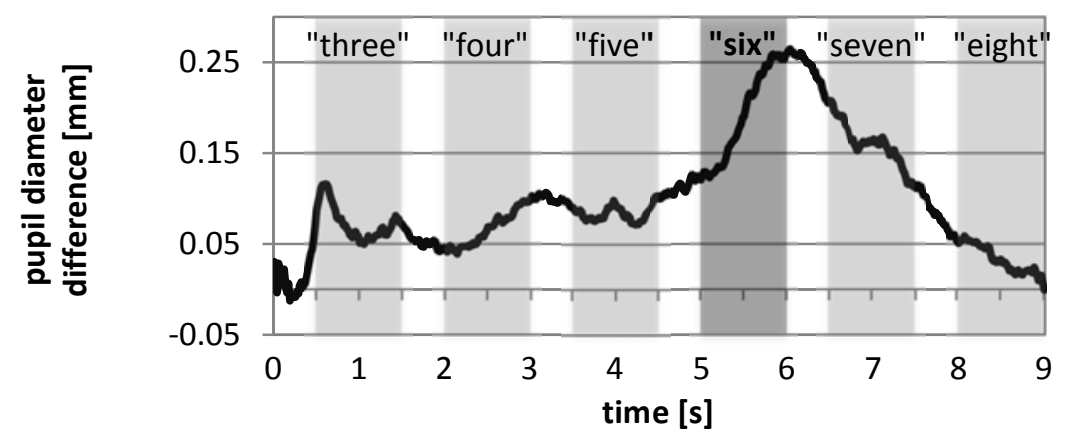

Figure 10. Difference between Combined Task and predictor output reveals cognitive load peak at $t=6 \mathrm{~s}$

\section{CONCLUSION AND FUTURE WORK}

We found that it is possible to dissociate the effects of light and cognitive load on pupil diameter for subjects scanning static images in a driving simulator (H1), although this might be difficult when the pupil size is large (when subjects are looking at a dark spot in which case the need for light overwhelms the mental load effect). We also found that it is possible to create a simple light 
reflex predictor, which would allow estimation of workload (H2). We want to reiterate that our results are averages of many instances of pupil diameter changes, which is useful in offline analysis. However, a different approach is needed to estimate cognitive load in real-time.

More research is needed on determining the field of view diameter (in degrees) that affects the pupil light reflex the most. We also plan to explore situations involving simulated driving and thus more diverse lighting conditions. Finally, we plan to refine the predictor to adapt to each subject as well as to eventually allow real-time estimation of cognitive load.

\section{ACKNOWLEDGMENTS}

This work was supported by the US Department of Justice under grant 2006DDBXK099.

\section{REFERENCES}

Asano S., Yasuike I., Nakayama M. \& Shimizu Y. (1994). A Pupil Reaction Model with Neural Network for Brightness Change. IEICE Transactions, 794-801, 77(5).

Beatty, J. (1982). Task-Evoked Pupillary Responses, Processing Load, and the Structure of Processing Resources. Psychological Bulletin, 276-292, 91(2).

Klingner, J., Kumar, R. \& Hanrahan, P. (2008). Measuring the Task-Evoked Pupillary Response with a Remote Eye Tracker. Proc. of Eye Tracking Research and Applications.

Palinko O., Kun A.L., Shyrokov A. \& Heeman P. (2010). Estimating Cognitive Load Using Remote Eye Tracking in a Driving Simulator. Proceedings of ETRA.

Pomplun, M. \& Sunkara, S. (2003). Pupil Dilation as an Indicator of Cognitive Workload in Human-Computer Interaction. Proceedings of the International Conference on HCI.

Van Orden, K.F., Limbert, W., Makeig, S. \& Jung, T. (2001). Eye Activity Correlates of Workload during a Visuospatial Memory Task. Human Factors, 111-121, 43(1). 